Diabetologe 2007 · 3:446-451

DOI 10.1007/s11428-007-0173-8

Online publiziert: 22. Oktober 2007

(c) Springer Medizin Verlag 2007
J. Nussberger

Département de médecine interne, Centre hospitalier universitaire vaudois, Lausanne

\section{Direkte Reninhemmer oder Kirene}

renzellen (• Abb. 2). Die hervorragende Bedeutung des RAAS und des Ang II für die Steuerung des Herz-Kreislauf-Gleichgewichts war bereits in den 1970er-Jahren bekannt [1]. Die Hemmung des Schrittmacherenzyms Renin versprach deshalb einen nachhaltigen Behandlungserfolg mindestens in jenen Fällen, wo zu viel Ang-II-Wirkung krank machte, v. a. bei gewissen Hypertonieformen [2]. Man wollte dem Ang II entgegenwirken, indem man seine Entstehung drosselte.

\section{Entwicklung der Reninhemmer}

Das Prinzip der Reninhemmung zur Hypertoniebehandlung ist alt, hat sich aber seit der Mitte des letzten Jahrhunderts nicht durchgesetzt, weil schlechte wie gute Reninantikörper $[3,4]$ ebenso intravenös verabreicht werden mussten wie die ersten peptidischen Antirenine [5, 6], weil die Reninhemmer zu schwach oder zu teuer in der Herstellung waren oder weil sie, oral verabreicht, nur im Promillebereich ins Blut übertraten, wie z. B. das Remikiren der Roche $[7,8]$. $\beta$-Blocker wirken als indirekte Reninhemmer, weil u. a. auch $\beta_{1}$ Rezeptoren die Reninausschüttung aus den Nieren vermitteln. Viel spezifischer - und mit dem Rasilez ${ }^{\bowtie}$ schließlich markttauglich - ist die direkte Reninhemmung mittels „falscher" Substrate, d. h. mit abgeän- derten Angiotensinogen- (Ang-N-)Bruchstücken, die anstelle des natürlichen Reninsubstrates Ang-N das katalytische Zentrum des Reninenzyms „verstopfen“ und damit die Reninaktivität und Ang-II-Bildung behindern (• Abb. 3).

Der Weg vom ersten klinisch getesteten Reninhemmer zum Rasilez ${ }^{\circledast}$ hat rund 20 Jahre gedauert $[5,9]$. Mehr als ein Dutzend meistens namhafter Pharmafirmen hat in den vergangenen Jahrzehnten substratanaloge Reninhemmer hergestellt und oft auch klinisch getestet. Fast alle haben ihre Bemühungen enttäuscht eingestellt: Methodische Mängel im Auswahlverfahren hatten ungeeignete Moleküle bis in die klinische Prüfung gebracht, wo sie dann jeweils einem Vergleichspräparat (ACE-Hemmer...) nicht standzuhalten vermochten. Immer wieder wurde daraus nicht richtigerweise gefolgert, dass das geprüfte Molekül ein schlechter Reninhemmer sei, sondern man schloss zu Unrecht, dass die Reninhemmung grundsätzlich nicht tauge - und gab auf.

Anders verhielt sich Novartis mit dem Rasilez ${ }^{\circledast}$ Synthetisiert als CGP 60’536 im Basler Mutterhaus [10] wurde dieser Reninhemmer für die Entwicklung an die neu gegründete Speedelpharma abgegeben und von dieser als Aliskiren erfolgreich klinisch getestet $[9,11,14]$, seine Synthese vereinfacht und schließlich wieder resorption aus dem Primärharn v. a. aus dem proximalen Nierentubulus, aber auch aus dem distalen Tubulus, u. a. indem es zur Aldosteronausschüttung aus den $\mathrm{Ne}$ bennieren führt. Zudem erhöht Ang II den zentralen Sympathikustonus. Ang II ist auch ein Wuchsfaktor, fördert den oxidativen Stress und ist beteiligt an der Entstehung der Atherosklerose. Den Regelkreis seiner eigenen Entstehung schließend, bremst Ang II die Ausschüttung des Renins aus den juxtaglomerulären Nie-
Abb. 1 - Strukturformel des Reninhemmers Aliskiren: Das substituierte Oktanamid vom Molekulargewicht 552 ist als Substratanalog ein potenter, oral wirksamer, kompetitiver Hemmer des humanen Renins. (Nach [9])

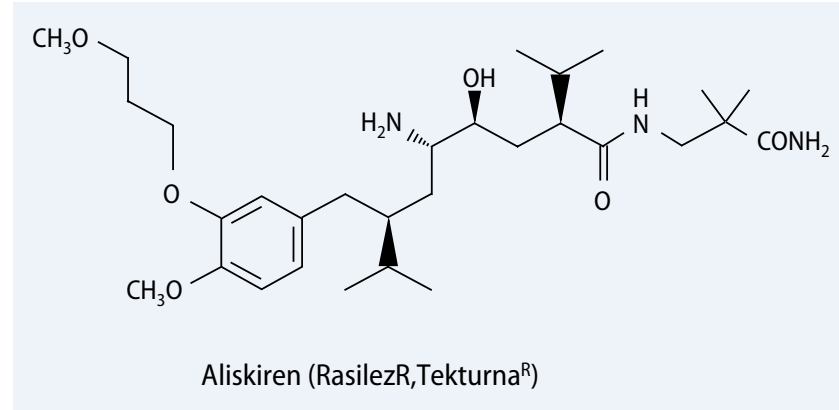


von Novartis für die klinischen Prüfungen der Phase III übernommen. Diese haben jetzt die Marktzulassung ermöglicht. Rasilez $z^{\circledast}$ ist ein lang wirksamer (Plasmahalbwertszeit $40 \mathrm{~h}$ ) kompetitiver Hemmer des menschlichen Renins $\left(\mathrm{IC}_{50}=10 \mathrm{nM}\right.$ in Plasma). Das substituierte Oktanamid hat ein Molekulargewicht von 552 (- Abb. 1). Geringe 3\% des Arzneimittels gelangen nach Tabletteneinnahme ins Blut. Dies genügt jedoch, um mit $150 \mathrm{mg}$ Rasilez $z^{\circledast}$ sämtliche Angiotensinspiegel und auch die Aldosteronausscheidung erheblich zu senken [9] und den Blutdruck mindestens ebenso herabzusetzen wie mit 100 mg Losartan (Cozaar $\left.{ }^{\circledR}\right), 150$ mg Irbes$\operatorname{artan}\left(\right.$ Aprovel $\left.^{\circledR}\right)$, $160 \mathrm{mg}$ Valsartan (Diovan) oder 5 mg Ramipril (Vesdil ${ }^{\circ}$ forte; $([11,12,13,14], \bullet$ Abb. 4). Kombinationsbehandlungen des Rasilez ${ }^{\oplus}$ mit diesen anderen Anti-RAAS-Blutdruckmitteln waren ebenso erfolgreich, wie wenn der Kalziumantagonist Amlodipin (Norvasc ${ }^{\circledast}$ ) oder das Diuretikum Hydrochlorthiazid $\left(\right.$ Esidrex $^{\oplus}$ ) mit dem Reninhemmer zusammen verabreicht wurde $[13,14,15]$ : Submaximale Dosen beider Stoffklassen erzielten jeweils additive Wirkungen und gelegentlich geringere Nebenwirkungen als der Partner des Reninhemmers allein [15].

\section{Indikationsbereich von Rasilez ${ }^{\circledR}$}

Bisher gut belegt ist die blutdrucksenkende Wirkung des Rasilez ${ }^{\circledR}$ in mehreren Untersuchungen mit 24-h-Blutdruckmessungen: Bei essenzieller Hypertonie senken 300 mg Rasilez ${ }^{\oplus}$ in Monotherapie den Tagesblutdruck um $-11 /-7 \mathrm{mmHg}$, und in Zweierkombinationen mit verschiedenen anderen Antihypertensiva werden bis zu weiteren $-7 /-4 \mathrm{mmHg}$ erreicht $[11,13,14]$. Die Nebenwirkungsrate war sehr gering und jener der ARB vergleichbar. Tierexperimentelle Ergebnisse zeigen unter Rasilez ${ }^{\oplus}$ eine verminderte Albuminurie, Ab- nahme der Herzhypertrophie und Schutz vor Atherosklerose [17]. Klinische Studien über möglicherweise verbesserte Nierendurchblutung, Nierenschutz und weniger Proteinurie sowie die Wirkung bei Herzinsuffizienz sind im Gang. Erste Ergebnisse sind innerhalb Jahresfrist zu erwarten. Beim europäischen Kardiologenkongress in Wien wurde soeben über eine 12-wöchige placebokontrollierte Doppelblindstudie bei 302 Herzinsuffizienten berichtet, deren Standardbehandlung mit u. a. $\beta$-Blockern und ACE-Hemmern/Sartanen mit täglich 150 mg Aliskiren ergänzt wurde: Der Reninhemmer vermochte die Plasmaspiegel des natriuretischen Peptids (BNP) signifikant weiter zu senken, was eine günstige Wirkung bei Herzinsuffizienz verspricht (ALOFT-Studie). Die größeren Endpunktstudien sind angelaufen, werden aber noch mehrere Jahre dauern. Langzeitstudien mit anderen Antiangiotensinen (ACE-Hemmer, Angiotensinre-

\section{Hier steht eine Anzeige.}

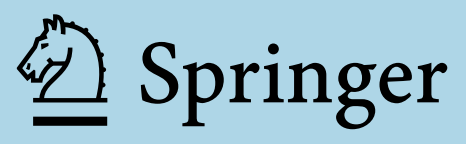




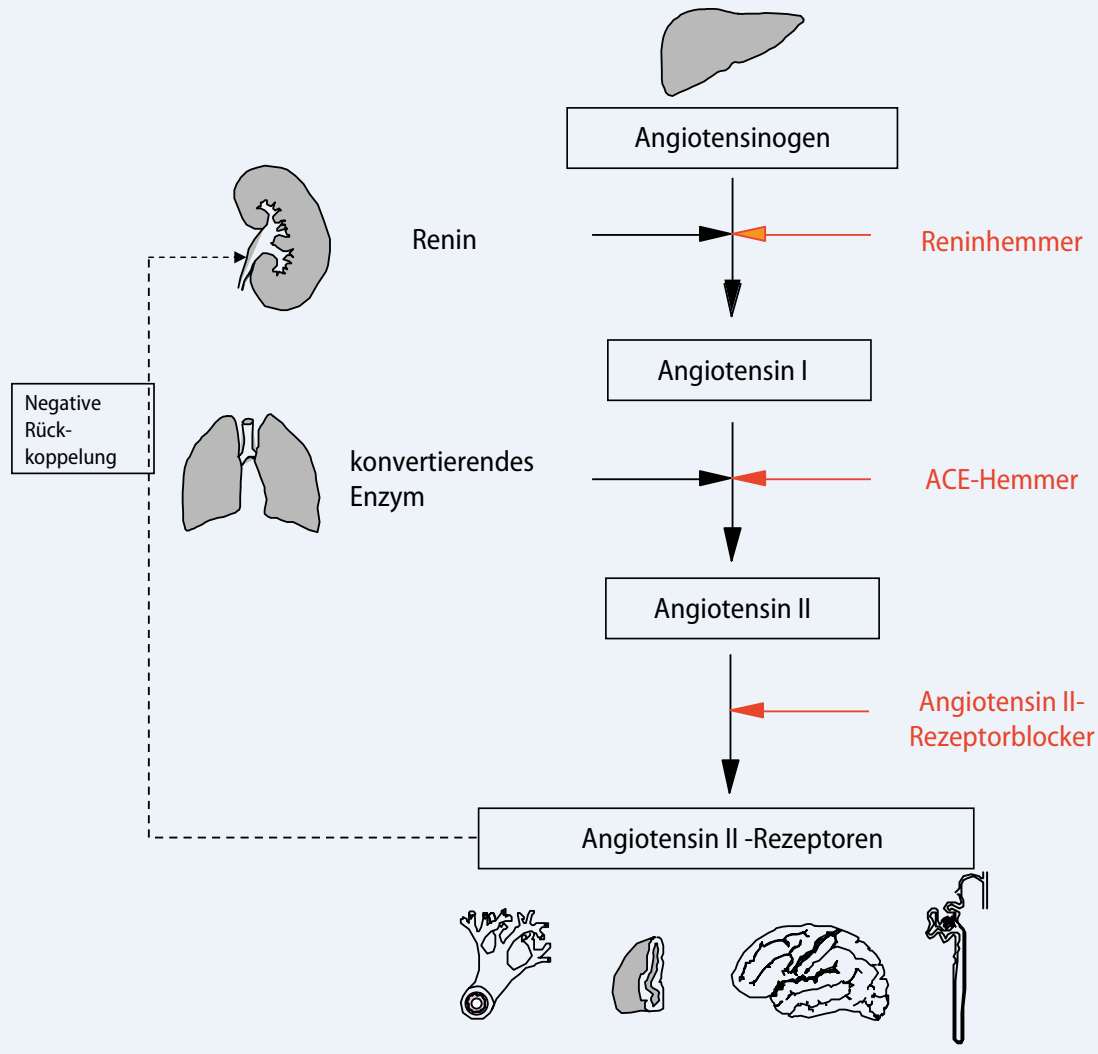

Abb. $2 \Delta$ Renin-Angiotensin-System und die verschiedenen Stufen zu seiner pharmakologischen Hemmung: Reninhemmung, ACE-Hemmung, Angiotensinrezeptorblockade

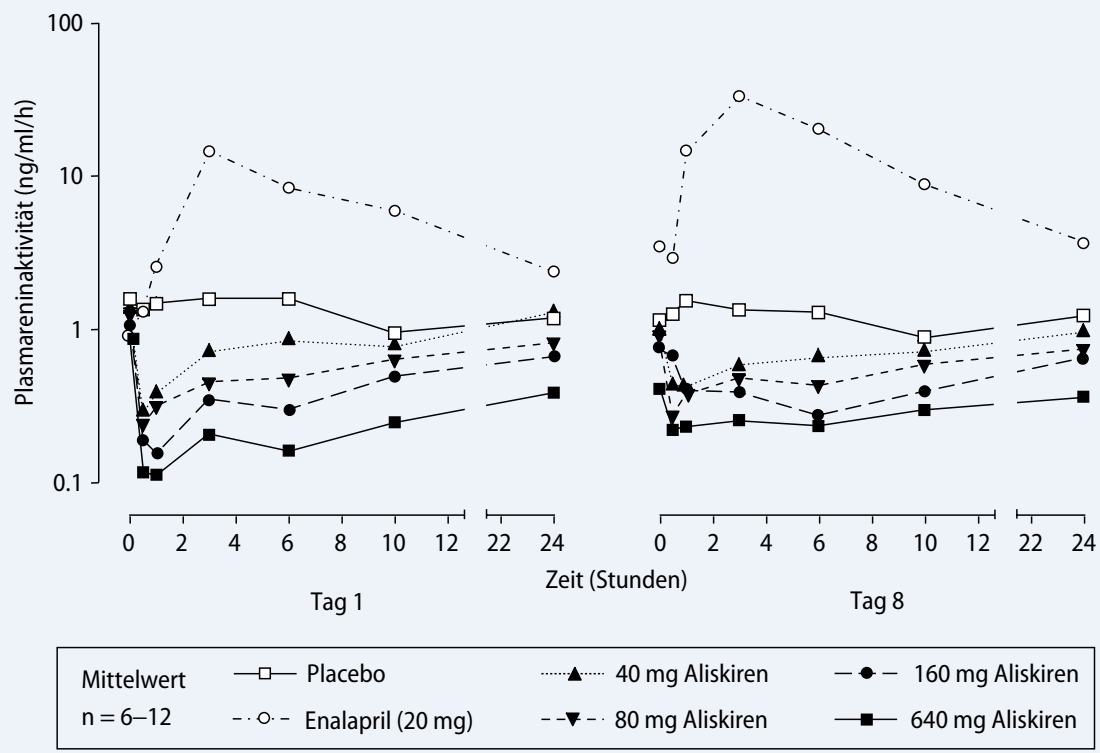

Abb. $3 \Delta$ 24-h-Profile der Plasmareninaktiviät (PRA) bei 9 gesunden Männern nach akuter (Tag 1) und andauernder (Tag 8) Hemmung des Renin-Angiotensin-Systems mit dem Reninhemmer Aliskiren und mit dem ACE-Hemmer Enalapril. Aliskiren senkt die PRA dosisabhängig. Enalapril steigert die PRA. (Nach [9])

zeptorblocker) haben eine Verminderung des Auftretens neuer Diabetesfälle ergeben. Das Zustandekommen dieser günstigen Wirkung ist noch nicht restlos geklärt, aber wenn die Ursache - wie vermutet eine Verminderung der Angiotensin-IISignale ist, müssten auch Reninhemmer Diabetesfälle verhindern helfen.
Wie die ACE-Inhibitoren (ACEI) und die Angiotensinrezeptorblocker (ARB) sind die direkten Renininhibitoren (DRI) unmittelbare Antiangiotensine, während $\beta$-Blocker und zentrale Sympathikolytika die Angiotensinspiegel indirekt absenken, indem sie einen Teil der Reninausschüttung aus den Nieren verhindern. Andere Antihypertensiva (mit Ausnahme der $\alpha$ Blocker) werden in ihrer Wirkung immer mehr oder weniger begrenzt, weil sie kompensatorisch eine Reninausschüttung und ungebremste Ang-II-Vermehrung hervorrufen (Kalziumantagonisten, Diuretika, direkte Vasodilatoren). Auch die DRI verstärken die Reninausschüttung, aber die Reninaktivität und die Angiotensinbildung steigen nicht an [18]. Für Rasilez ${ }^{\circledR}$ mit seiner langen Halbwertszeit gilt dies ganz besonders. Letztere dürfte zusammen mit der guten Verträglichkeit dem Rasilez ${ }^{\circledR}$ eine erhöhte Behandlungstreue (Patientencompliance) eintragen. Seine Nebenwirkungen sind jenen der ARB vergleichbar und bisher kaum anders als jene von Placebobehandlungen $[11,12,13,14]$. Überdosierungen können zu Darmreizungen mit Diarrhö führen. Im Gegensatz zu den ARB senken DRI sämtliche Angiotensinspiegel, was unerwünschte ARB-Wirkungen über andere Angiotensine und deren Rezeptoren ausschließt (z. B. keine PAI-1-Stimulierung über $\mathrm{AT}_{4}$-Rezeptor mit potenzieller Thrombose). Im Gegensatz zu den DRI, die spezifisch die Schrittmacherenzymreaktion des RAAS hemmen [Renin setzt aus mikromolarem $\left(10^{-6} \mathrm{M}\right)$ Ang-N das Ang I frei], hemmen ACEI eine wenig spezifische Konvertase, die aus picomolarem $\left(10^{-12} \mathrm{M}\right)$ Ang I das wirksame Ang II freisetzt. Die gleiche Konvertase baut auch Substanz P und Bradykinin ab. ACEI führen deshalb zur Anhäufung dieser Peptide und verursachen entsprechend in 10\% der Behandlungen Husten und in 1\% der jährlichen ACEI-Therapien potenziell tödliche Angioödeme (Larynxödem!). Falls ACEI über die Bradykininanhäufung günstig z. B. auf die Koronargefäße wirken, würde diese Wirkung den DRI abgehen. Unter ACEHemmung versiegt die Ang-II-Produktion nie vollständig, weil die Ang-I-Spiegel stark ansteigen und zudem andere Enzyme wie die Chymase ebenfalls Ang I in Ang II umwandeln können. Diese Umgehungsreaktion bleibt unter DRI wirkungslos. DRI 
Hier steht eine Anzeige.

第 Springer 
Diabetologe 2007·3:446-451 DOI 10.1007/s11428-007-0173-8

○) Springer Medizin Verlag 2007

\section{J. Nussberger \\ Direkte Reninhemmer oder Kirene}

\section{Zusammenfassung}

Die direkten Reninhemmer (DRI) bilden eine neue Klasse blutdrucksenkender Arzneimittel. Wie die ACE-Hemmer und die Angiotensinrezeptorblocker (ARB) dämpfen sie das Renin-Angiotensin-Aldosteronsystem (RAAS). Als gut verträgliche und hochspezifische Therapeutika senken sie die Plasmaspiegel sämtlicher Angiotensine und dürften in Zukunft erfolgreich bei Herz-Kreislauf- und Nierenkrankheiten eingesetzt werden. DRI verursachen weder Husten noch Angioödeme, was sie vor den ACE-Hemmern auszeichnet. Auch metabolische Nebenwirkungen fehlen (keine Dyslipidämie, Hyperurikämie, Diabetes...).

\section{Direct renin inhibitors or kirens}

\begin{abstract}
Direct renin inhibitors (DRIs) are a new class of antihypertensive drugs. DRIs reduce the activity of the renin-angiotensin-aldosterone system (RAAS) and are therefore comparable to angiotensin receptor blockers (ARBs) and angiotensin converting enzyme inhibitors (ACEIs). In contrast to the latter, however, they decrease the concentrations of all angiotensins and do not cause cough or angioedema. DRIs are highly specific and very well tolerated drugs. They are primarily used as antihypertensives, but promise to be useful for organ protection in other cardiovascular and renal diseases. DRIs are an alternative to oth-
\end{abstract}

DRI können als Alternative oder als Kombinationspartner zu anderen RAAS-Blockern (ARB, ACE-Hemmern, $\beta$-Blockern, Aldosteronantagonisten), Kalziumantagonisten oder Diuretika werden verwendet. Aliskiren/Rasilez $z^{\circledast}$ ist ein erster lang wirksamer und gut verträglicher Reninhemmer, der als Monotherapie oder in Kombination mit anderen Antihypertensiva eingesetzt wird.

\section{Schlüsselwörter}

Bluthochdruck · ACE-Inhibitoren · Angiotensin · Angiotensinrezeptorblocker · Blutdrucksenkende Arzneimittel

er RAAS-blocking agents (ARBs, ACEls, beta blockers, aldosterone antagonists), calcium channel blockers, and diuretics or can be used in combination with them. Aliskiren/ Rasilez ${ }^{\circledR}$ is the first orally active DRI to provide long-acting antihypertensive effects and an excellent tolerability profile; it has been successfully used as a monotherapy and in combination with other antihypertensive drugs.

\section{Keywords}

Hypertension · ACE inhibitors · Angiotensin . Angiotensin receptor blocker · Antihypertensive drug neigen naturgemäß als RAAS-Hemmer zu leichtem Anheben des Serumkaliumspiegels, was v. a. bei Niereninsuffizienz beachtet werden muss und was sie zu geeigneten Partnern kaliuretischer Diuretika macht. DRI zeigen aber keine metabolischen Wirkungen, was sie u. a. im Gegensatz zu $\beta$ Blockern (diabetogen, hypoglykämiemaskierend, Alpträume, kalte Extremitäten...) und Diuretika (diabetogen, Dyslipidämie, Gicht, Hypokaliämie...) auszeichnet. Auch die störenden peripheren Ödeme, die unter Kalziumantagonisten auftreten, werden unter DRI nicht beobachtet. Im Gegenteil, die Ödeme gehen unter Kombinationsbehandlung mit Kalziumantagonist und Rasilez ${ }^{\circledast}$ zurück [15].

\section{(D) Reninhemmer senken die Plasmaspiegel sämtlicher Angiotensine und dürften in Zukunft bei Herz-Kreislauf- und Nierenkrankheiten eingesetzt werden}

Die direkten Reninhemmer (DRI) bilden eine neue Klasse der Antihypertensiva. Als jüngste Vertreter der Antiangiotensine bestätigen sie auch die älteste Arbeithypothese der RAAS-Blockade, dass den Blutdruck senkt, wer Angiotensin II senkt. Wie die ACE-Hemmer und die ARB dämpfen die Reninhemmer das RAAS. Als gut verträgliche und hochspezifische Therapeutika senken sie die Plasmaspiegel sämtlicher Angiotensine und dürften in Zukunft erfolgreich bei HerzKreislauf- und Nierenkrankheiten eingesetzt werden. DRI dürften als Alternative oder als Kombinationspartner zu anderen RAAS-Blockern (ARB, ACEI, $\beta$-Blockern, Aldosteronantagonisten) verwendet werden. Rasilez ${ }^{\circledast}$ ist ein erster lang wirksamer und gut verträglicher Reninhemmer, der als Monotherapie oder in Kombination mit Kalziumantagonisten oder Diuretika ein Erfolgsrezept darstellen könnte.

\section{Fazit für die Praxis}

Novartis hat mit dem kassenzulässigen Rasilez ${ }^{\circledast} /$ Aliskiren eine neue Klasse blutdrucksenkender Arzneimittel eingeführt: die direkten Reninhemmer (DRI). Bisher wurden rund 10.000 Patienten mit Rasilez ${ }^{\circledast}$ behandelt. Erwartungsgemäß wirkt 


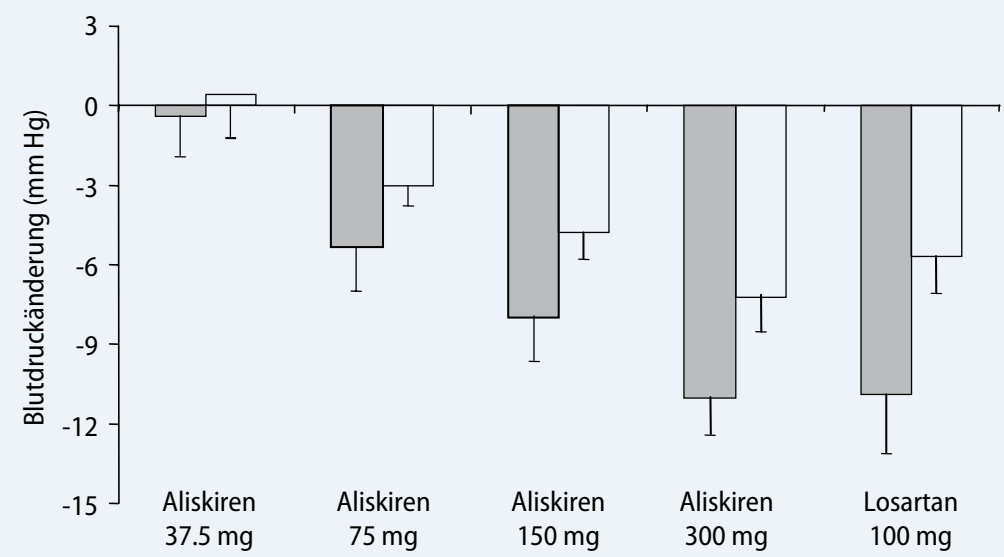

M-SEM, $\square$ Systolischer ambulatorischer Tagesblutdruck nach vier Behandlungswochen

$\mathrm{n}=36-41 \square$ Diastolischer ambulatorischer Tagesblutdruck nach vier Behandlungswochen

Abb. $4 \Delta$ Antihypertensive Wirkung des Reninhemmers Aliskiren und des Angiotensinrezeptorblockers Losartan bei Patienten mit essenzieller Hypertonie: mittlere Änderung des ambulatorischen systolischen und diastolischen Tagesblutdrucks nach 4 Wochen Behandlung mit Losartan und verschiedenen Dosen des oral wirksamen Reninhemmers Aliskiren. Die höheren Dosen des Reninhemmers senken den Blutdruck ebenso wie der Angiotensinantagonist. (Nach [11])

auch dieser neue Hemmer des Renin-Angiotensin-Aldosteron-Systems (RAAS) deutlich antihypertensiv. Er kann als Monotherapie oder in Kombination mit praktisch allen anderen Antihypertensiva eingesetzt werden. Seine sehr lange Wirkungsdauer dürfte diesem Reninhemmer gegenüber anderen RAAS-Blockern die Vorteile anhaltender Blutdrucksenkung und möglicherweise besseren Organschutzes verschaffen. Tierexperimente mit Aliskiren ergaben eine Abnahme von Albuminurie, Herzhypertrophie und Atherosklerose. Falls der Schutz anderer RAAS-Blocker (ACE-Hemmer, ARB) vor neu auftretendem Diabetes tatsächlich antiangiotensinbedingt ist, müssten auch DRI einen solchen Schutz gewähren. Entsprechende klinische Studien mit Rasilez ${ }^{\circledast} /$ Aliskiren fehlen noch.

Bezüglich Nebenwirkungen schneidet Rasilez $z^{\circledast}$ im Vergleich mit anderen Antihypertensiva sehr gut ab: Obwohl ähnlich wirkend wie die ACE-Hemmer (bremst die Bildung von Angiotensin II), verursacht Rasilez $z^{\circledast}$ als hochspezifisches Medikament deutlich weniger Nebenwirkungen als die ACE-Hemmer (keinen Husten, keine Angioödeme). Im Gegensatz zu den Angiotensinrezeptorblockern (ARB) senken die neuen DRI die Konzentrationen sämtlicher Angiotensine, was auch unerwünschte Wirkungen $z$. B. des prothrombotischen Angiotensins IV ausschließt. Die peripheren Ödeme der Kalziumantagonisten treten unter DRI nicht auf, und sie werden bei Kombinationsbehandlung vermindert. Rasilez $z^{\oplus}$ ist im Unterschied zu den Diuretika und $\beta$-Blockern nicht diabetogen und teilt auch deren übrige metabolischen Nebenwirkungen nicht. Hingegen neigen DRI wie die ACE-Hemmer und die ARB dazu, die Plasmakaliumspiegel leicht anzuheben, was $v$. a. bei verminderter Nierenfunktion berücksichtigt werden muss. Eine erfolgreiche Blutdruckeinstellung erfordert eine gute Behandlungstreue (Patientencompliance) und meistens eine Behandlung mit mehreren Medikamenten. Ein neues Antihypertensivum ist daher zu begrüßen, wenn es gut verträglich mit den meisten anderen Stoffklassen kombiniert werden kann und zudem sehr lange wirkt, sodass die Tabletteneinnahme auch einmal vergessen werden darf. Der Reninhemmer Rasilez ${ }^{\circledast}$ erfüllt diese Bedingungen.

\section{Korrespondenzadresse}

Prof. J. Nussberger

Département de médecine interne, Centre hospitalier universitaire vaudois 1011 Lausanne

Schweiz

Juerg.Nussberger@chuv.ch
Interessenkonflikt. Der korrespondierende Autor weist auf folgende Beziehung hin: Forschungszusammenarbeit mit der Fa. Novartis, Basel.

\section{Literatur}

1. Oparil S, Haber E (1974) The renin-angiotensin system. N Engl J Med 291: 389-401

2. Bühler FR, Laragh JH, Vaughan ED et al. (1973) Antihypertensive effect of propranolol: specific anti-renin responses in high and normal renin forms of essential, renal, renovascular and malignant hypertension. Am J Cardiol 32: 511-522

3. Johnson CA, Wakerlin GE (1940) Antiserum for renin. Proc Soc Exp Biol Med 44: 277-281

4. Dzau V, Kopelman RI, Barger AC et al. (1984) Comparison of renin-specific $\lg \mathrm{G}$ and antibody fragments in studies of blood pressure regulation. Am J Physiol 246: H404-H409 and 247: XXXII

5. Zusman RM, Burton J, Christensen D et al. (1983) Hemodynamic effects of a competitive renin inhibitory peptide in humans. Evidence for multiple mechanisms of action. Trans Assoc Am Physicians 96: 365-374

6. Weber AM, Neutel JM, Essinger I et al. (1990) Assessment of renin dependency of hypertension with a dipeptide renin inhibitor. Circulation 81: 1768-1774

7. Camenzind E, Nussberger J, Juillerat L et al. (1991) Effect of the renin response during renin inhibition: Oral RO 42-5892 in normal humans. J Cardiovasc Pharmacol 18: 299-307

8. Van den Meiracker AH, Admiraal PJJ, Man in, t Veld AJ et al. (1990) Prolonged blood pressure reduction by orally active renin inhibitor RO 42-5892 in essential hypertension. Br Med J 301: 205-210

9. Nussberger J, Wuerzner G, Jensen C, Brunner HR (2002) Angiotensin Il suppression in humans by the orally active renin inhibitor aliskiren (SPP100). Hypertension 39: e1-e8

10. Bühlmayer $P$, Caselli W, Fuhrer W et al. (1988) Synthesis and biological activity of some transition-state inhibitors of human renin. J Med Chem 31: 1839-1846

11. Stanton A, Jensen C, Nussberger J, O'Brien E (2003) Blood pressure lowering in essential hypertension with an oral renin inhibitor, aliskiren. Hypertension 42: 1137-1143

12. Gradman A, Schmieder RE, Lins RL et al. (2005) Aliskiren, a novel orally effective renin inhibitor, provides dose-dependent antihypertensive efficacy and placebo-like tolerability in hypertensive patients. Circulation 111: 1012-1018

13. Oparil S, Yarows SA, Patel S et al. (2007) Efficacy and safety of combined use of aliskiren and valsartan in patients with hypertension: a randomized, doubleblind trial. Lancet 370: 221-229

14. O'Brien E, Barton J, Nussberger J et al. (2007) Aliskiren reduces blood pressure and suppresses plasma renin acivity in combination with a thiazide duretic, an angiotensin-converting enzyme inhibitor, or an angiotensin receptor blocker. Hypertension 49: 1-9

15. Munger MA, Drummond W, Essop MR et al. (2007) Aliskiren as add-on to amlodipine provides significant additional blood pressure lowering without increased oedema associated with doubling the amlodipine dose. Eur Heart J 27 (Suppl 1): 177 (P784)

16. O'Brien E, Barton J, Nussberger J et al. (2007) Aliskiren reduces blood pressure and suppresses plasma renin acivity in combination with a thiazide duretic, an angiotensin-converting enzyme inhibitor, or an angiotensin receptor blocker. Hypertension 49: 1-9

17. Pilz B, Shagdarsuren E, Wellner M et al. (2005) Aliskiren, a human renin inhibitor, ameliorates cardiac and renal damage in double-transgenic rats. Hypertension 46: $569-576$

18. Nussberger J (2005) Renin inhibitors. In: Oparil S, Weber MA (eds) Hypertension, 2nd edn. Elsevier Saunders, Philadelphia, pp 754-764 